\title{
EMPRENDIMIENTOS PERIODÍSTICOS: UNA SALIDA A LA CRISIS DE LAS EMPRESAS INFORMATIVAS
}

\section{JOURNALISM ENTREPRENEURS: AN OUTPUT TO THE CRISIS OF THE MEDIA COMPANIES}

\section{ANA GABRIELA DÁVILA J.$^{1}$}

Recibido: 4 de enero de 2017 Aceptado: 6 de marzo de 2017

\footnotetext{
${ }^{1}$ Pontificia Universidad Católica del Ecuador, Facultad de Comunicación, Lingüística y Literatura, Quito, Ecuador (anagadavila@gmail.com).
} 



\section{EMPRENDIMIENTOS PERIODÍSTICOS: UNA SALIDA A LA CRISIS DE LAS EMPRESAS INFORMATIVAS}

\section{JOURNALISM ENTREPRENEURS: AN OUTPUT TO THE CRISIS OF THE MEDIA COMPANIES}

ANA GABRIELA DÁVILA J.

PALABRAS CLAVE: crisis de los medios, emprendimientos periodísticos, contenidos periodísticos, modelos de negocio

KEY WORDS: media crisis, journalism enterpreneurs, journalism contents, business models

\section{RESUMEN}

La crisis que, actualmente, viven las empresas informativas en todo el mundo, producto de la revolución digital y de la crisis económica mundial, ha ocasionado cambios sustanciales en varios aspectos inherentes a este tipo de organizaciones. Uno de ellos está relacionado con los ingresos económicos que reciben estas empresas y otro con la transformación de las funciones que de- ben realizar los periodistas que laboran en ellas. Esto ha afectado a la plantilla de trabajadores de los medios, misma que cada vez va reduciéndose.

Si bien en el contexto internacional se aduce a la revolución digital y a la crisis económica, como las principales causas de esta situación, en el contexto nacional existen otros factores como la relación medios-gobierno que también 
ha contribuido para que el trabajo formal en medios de comunicación, sobre todo en prensa escrita, se torne más complejo.

Producto de esta realidad han surgido nuevas formas de hacer periodismo, iniciativas que plantean un periodismo más independiente y menos anclado a las estructuras formales de los medios de comunicación tradicionales. A estos nuevos proyectos se les ha denominado emprendimientos periodísticos.
Este artículo expondrá elementos que muestren como se está desarrollando el emprendimiento periodístico en la Región y en el país, a través de la identificación de proyectos concretos y del análisis de los factores que han influido para el aparecimiento de esta tendencia. Además, mostrará los modelos de negocio que estos nuevos medios están aplicando para rentabilizar su actividad.

\section{ABSTRACT}

The current crisis in information companies around the world, as a result of the digital revolution and the global economic crisis, has brought substantial changes in several aspects inherent in this type of organization. One of them is related to the economic income that these companies receive and another to the transformation of the functions that must be done by the journalists who work in them. This has affected the workforce of the media, which is declining more and more.

Although the digital revolution and the economic crisis are the main causes of this situation in the international context, in the national context there are also other factors, such as the media-government relationship that has contributed to the fact that formal work in media Communication, especially in the written press, becomes more complex.

As a result of this reality, new forms of journalism have emerged, initiatives that pose a more independent journalism and less anchored to the formal structures of traditional media. These new projects have been called Entrepreneurial journalism.

This article will expose elements that show how Entrepreneurial journalism is developing in the region and in the country through the identification of concrete projects and the analysis of the factors that have influenced the emergence of this tendency. In addition, it will show the business models that these new media are applying to monetize their activity. 


\section{INTRODUCCIÓN}

La publicidad ha sido, históricamente, el sustento económico de los medios de comunicación, sin embargo, en las primeras empresas informativas, el concepto de rentabilidad empresarial no estaba presente. Desde finales del siglo XVII y hasta casi la mitad del siglo XIX, la prensa se caracterizó por posicionar ideas y defender intereses personales de las élites políticas, literarias y culturales, sin buscar un fin económico, sino más bien la propagación de una ideología. (Viloria, Alonso 2005).

El siglo XVIII se caracteriza por ser un período de transformación de los medios de comunicación, en el que se comienzan a perfilar los modelos de negocio y de gestión, que con sus correspondientes adaptaciones, conocemos hoy en día. El concepto de rentabilidad económica y de función social, empieza a ser introducido en las incipientes empresas informativas de la época. (Viloria, Alonso 2005).

El primer registro que se tiene de la presencia de publicidad en la prensa escrita, como estrategia para conseguir réditos económicos, señala al diario inglés The Spectator, en 1711, como el pionero en establecer un mecanismo que permitía que, a través de la venta de publicidad, se abaraten los costos de cada edición, al ser los anunciantes quienes los financiaban. De este modo, surge el concepto moderno de tarifa publicitaria, a través del cual un medio cotiza el valor de su espacio publicitario en función de su tiraje o rating.

A partir de entonces, la publicidad ha sido inherente al desarrollo y subsistencia de los medios de comunicación, ha sido la fuente principal de sus ingresos y el elemento fundamental de su actividad comercial.

La crisis económica mundial y la revolución digital, principalmente, han reducido los ingresos por publicidad en los medios de comunicación tradicionales, en todo el mundo. Los nuevos soportes publicitarios digitales resultan atractivos para las marcas, no solo porque a través de estos se conectan de mejor manera con sus consumidores, por el tipo de piezas publicitarias que pueden ofrecerles, sino también, porque es en el entorno digital, donde, actualmente, se encuentran la mayoría de ellos. Otra razón por la que las marcas están prefiriendo la publicidad digital, es el costo de inversión, el mismo que resulta relativamente barato, en comparación a la inversión en medios tradicionales.

En una época de crisis económica global, en la que uno de sus síntomas se 
manifiesta con la reducción de los presupuestos para publicidad en todas las empresas, y en la que es posible desarrollar estrategias publicitarias digitales a bajo costo, el camino más viable resulta abandonar la publicidad en los medios tradicionales y mudarse a la web.

En el país existen pocos estudios sobre inversión publicitaria digital, sin embargo, por su trascendencia, al ser uno de los primeros en desarrollarse, cabe referirse al realizado por lab Ecuador, Asociación Internacional sin fines de lucro, enfocada a maximizar la efectividad de la publicidad interactiva. En este informe, correspondiente al 2013, se señala que la inversión publicitaria en Internet al cierre de ese año fue de USD 7411 025, de los cuales un 55\% fue destinado a display; un 12\% a móvil y un 33\% a administración de redes sociales. Además, la inversión total del segundo semestre de ese año fue USD 4 076 064,18 y presentó un crecimiento del 22\%, frente al primer semestre del año anterior que fue de USD 3334 961,60. Redacción Quito (2015, 22 de marzo) La inversión publicitaria decae en medio de la irrupción digital. Revista Lideres. Recuperado de http://www.revistalideres.ec/lideres/inversion-publicitaria-digital-ecuador.html

Según un informe de Infomedia, empresa especializada en auditoría y estadísticas publicitarias, la inversión publicitaria en los medios tradicionales ecuatorianos decreció un 13,9\% en octubre del 2015, con respecto al mismo mes del 2014. Redacción Quito (2016, 5 de enero) La publicidad cae el 13,9\% en los medios tradicionales, pero aumenta en sitios web. Diario El Telégrafo.

Recuperado de http://www.eltelegrafo.com.ec/noticias/medios/1/la-publicidad-cae-el-13-9-en-los-medios-tradicionales-pero-aumenta-en-sitios-web

Esta realidad no solo es nacional, sino mundial. De acuerdo con el informe del Centro de Investigaciones PEW, especializado en el análisis de problemáticas y tendencias presentes en Estados Unidos, publicado en abril del 2015, sobre el estado de los medios de comunicación, desde el 2011 la publicidad digital ha ganado terreno, frente a la tradicional. Eso se demuestra en un aumento de $\$ 19$ mil millones en la inversión de publicidad digital en 4 años y en la pérdida de $\$ 3$ mil millones para los medios tradicionales, por el mismo rubro, en el mismo período. Aunque el informe está enfocado en Estados Unidos, permite hacer un balance global, tomando en cuenta que la mayoría de las grandes empresas de comunicación, publicidad, entretenimiento y de negocios se encuentra en este país. Redacción Quito (2016, 5 de enero) La publicidad cae el 13,9\% en los medios tradicionales, pero aumenta en sitios web. Diario El Telégrafo. Recuperado de http://www.eltelegrafo.com.ec/noticias/ 
medios/1/la-publicidad-cae-el-13-9-enlos-medios-tradicionales-pero-aumentaen-sitios-web

En el Ecuador, la reducción de los ingresos publicitarios, no ha sido la única dificultad que han debido sortear los medios de comunicación. Desde el 2007, año en que Rafael Correa asumió la presidencia del Ecuador, se han generado varios hechos que han marcado la relación prensa-poder, la misma que se ha desgastado al punto de afectar la estabilidad de las empresas informativas. Juicios contra medios y periodistas, la creación de la Ley de Comunicación, la conformación de instancias reguladoras de la comunicación e información como la Secretaría de Comunicación (SECOM), la Superintendencia de la Información y Comunicación (SUPERCOM) y el Consejo de Regulación y Desarrollo de la Comunicación e Información (CORDICOM) y las permanentes acusaciones por parte del Presidente de la República en contra de los medios de comunicación, cuestionando su manejo editorial y su función social.

Según un informe de Fundamedios, el 2015 fue uno de los años más críticos para el ejercicio de la libertad de expresión y, en particular, para el trabajo de los medios de comunicación. Desde enero a diciembre, esta entidad contabilizó 377 agresiones, registrando un aumento del $44 \%$ respecto al 2014, que cerró con 254 agresiones. Según este informe, las agresiones se elevan a un total de 1407, desde que se inició el monitoreo en el 2008. Portal Juicio Crudo (2016, 11 de enero) Con un récord negativo de 377 agresiones, 2015 cierra como el peor año para la libertad de expresión en Ecuador. Quito. Recuperado de http://www. juiciocrudo.com/articulo/con-un-record-negativo-de-377-agresiones-2015cierra-como-el-peor-ano-para-la-libertad-de-expresion-en-el-ecuador/5206

A esta realidad propia del contexto nacional, se suma otra del contexto global, la transformación del trabajo periodístico, producto de la revolución digital. Las rutinas de trabajo han cambiado en función de generar productos informativos multimediales, interactivos, hipertextuales y multiplataforma, lo que exige que los periodistas tengan nuevos conocimientos relacionados con las herramientas digitales y las nuevas narrativas periodísticas. Los medios de comunicación necesitan periodistas polifuncionales, que cumplan con varias tareas a la vez, y que tengan la predisposición de trabajar al ritmo vertiginoso de las transformaciones tecnológicas. Precisan de profesionales abiertos al cambio y a la innovación y, sobre todo, que hayan asimilado el hecho de estar inmersos en una nueva era del periodismo. Esto ha generado que muchas empresas periodísticas prescindan de gran parte de su 
plantilla, ya sea porque han reestructurado los puestos de trabajo y ahora las tareas que hacían tres periodistas, las hace uno, o porque, simplemente, los perfiles ya no se ajustan a lo que los medios necesitan; más amplios y versátiles que incluyan saberes de otras ramas del conocimiento. La falta de ingresos publicitarios ha agudizado aún más esta situación, por lo que muchos medios han optado por reducir sus gastos, despidiendo personal.

Según la última Encuesta Nacional de Empleo, Desempleo y Subempleo del INEC, en septiembre de 2016 la tasa de desempleo se registró en un 5,2\% a nivel nacional. Portal del Instituto Ecuatoriano de Estadísticas y Censos INEC (2016, 17 de octubre) INEC publica cifras del mercado laboral de septiembre 2016. Quito. Recuperado de http://www. ecuadorencifras.gob.ec/inec-publica-cifras-del-mercado-laboral-de-septiembre-2016/. Mientras que en el tercer trimestre de 2015 fue de 4,28\%. Un estudio de la Consultora AMS Andean Ecuador señaló que en el primer trimestre de este mismo año se desvinculó entre el 7,5\% y el 30,7 del personal de empresas de distintos sectores. Los medios de comunicación no fueron la excepción, el Grupo "El Comercio", por ejemplo, ajustó su nómina en un 20\%, a lo largo del 2015. Enríquez, Carolina (2015, 2 de diciembre) Los puestos de trabajo se reducen en una coyuntura económica difícil. "El Comercio". Recuperado de http://www. elcomercio.com/actualidad/desempleo-trabajadores-crisis-economia-empresas.html

Este año fue particularmente complicado para los medios, no solo Grupo El Comercio hizo cambios en su plantilla, TC Televisión desvinculó a 65 empleados, 50 de Guayaquil y 15 de Quito. Gama Tv, por su parte, despidió a 30 personas de distintas áreas, entre ellas periodistas. En el 2014, en cambio, fue Ecuavisa el canal que hizo recortes: cerca de 60 personas salieron de este medio, entre ellas personal del área de noticias y producción. El reconocido periodista Félix Narváez, con 20 años de trabajo en ese canal, estuvo entre los despedidos. Redacción Quito (2014, 17 de septiembre) Televisora privada Ecuavisa despide a 40 trabajadores para "bajar costos". Agencia Pública de Noticias del Ecuador y Sudamérica Andes. Recuperado de http://www.andes.info.ec/es/noticias/televisora-privada-ecuavisa-despide-40-trabajadores-bajar-costos.html. A estos se suman las 172 personas que tuvieron que ser liquidadas tras el cierre de diario "Hoy", también ocurrido en el 2014, y las cuarenta personas que fueron despedidas de la editorial Televisa, donde se elaboraba revista Caras y Vanidades, entre otras, misma que cerró sus oficinas en el país. 
La crisis de las empresas informativas, producto de todo lo señalado, ha abierto una nueva vía para hacer periodismo: el emprendimiento, mismo que es visto no solo como una alternativa para enfrentar la crisis, sino que evidencia que estamos ante una nueva era de la profesión.

\section{Emprendimientos periodísticos en Latinoamérica}

Latinoamérica ha sido, tradicionalmente, una región azotada por problemáticas sociales como el narcotráfico, la guerrilla, la corrupción, las cuales han sido tratadas por los medios tradicionales, muchas veces con perspectivas sesgadas y en función de salvaguardar los intereses de los grupos mediáticos, históricamente, relacionados con el poder.

En esta región del mundo, los emprendimientos periodísticos no solamente se han configurado como una salida a la crisis de los medios, sino como una manera de erigir un espacio desde el que se hace un periodismo independiente, cuestionador, riguroso, casi de denuncia, en algunos casos, cuyo interés es transparentar aquello que permanece oculto y tratar aquello que le interesa al ciudadano común, a las pequeñas comunidades, aquello que no está presente en los medios tradicionales. También lo que les interesa a las nuevas generaciones de jóvenes. "Los medios tradicio- nales generan información sobre la clase política y para la clase política; hay poca cobertura de los temas ciudadanos, de la sociedad civil, que son los temas que más consumen los jóvenes". Quesada, Juan Diego (2013, 1 de marzo) El "boom" de la prensa digital latinoamericana. Diario El País. Recuperado de http://sociedad.elpais.com/sociedad/2013/03/01/ actualidad/1362165444 533165.html

Estos emprendimientos representan una forma fresca y genuina de hacer buen periodismo, que, al mismo tiempo, reivindica la función social de la profesión, la que muchas veces se ha diluido en la dinámica habitual de las empresas informativas tradicionales, por los varios intereses que las rodean. Además, representan, de alguna forma, una reconexión con lo humano del oficio periodístico.

El caso emblemático de esta tendencia, por ser el primer periódico nativo digital de Latinoamérica, es el periódico salvadoreño "El Faro", especializado en investigar temas relacionados con el poder político. Tiene 18 años de vida, a lo largo de los cuales ha sido muy difícil consolidar el proyecto desde el ámbito económico, aun cuando en lo periodístico, se ha convertido en un referente de un periodismo independiente y audaz. Durante su participación en el Congreso de Periodismo Digital, organizado en Quito por Fundamedios y la Universidad 
Internacional del Ecuador, en el 2015, Carlos Dada, fundador de "El Faro", dijo que durante aproximadamente quince años, el periódico no generó ganancias económicas. Señaló que desde el principio del proyecto, la mayoría de los periodistas que colaboraban en él, tenían otros trabajos que eran su sustento económico. Sin embargo, a lo largo de este mismo tiempo, el periódico logró importantes reconocimientos internacionales que destacan su excelencia periodística. En el 2011 obtuvo el premio María Moors-Cabot otorgado por la Universidad de Columbia. En el mismo año ganó el Premio Ortega y Gasset en Periodismo. En el 2013 fue finalista en la primera edición del premio "Gabriel García Márquez de Periodismo", en la categoría Innovación y en el 2016 recibió un reconocimiento a la excelencia en la cuarta edición de este mismo premio. Quesada, Juan Diego (2013, 1 de marzo) El "boom" de la prensa digital latinoamericana. Diario El País. Recuperado http://sociedad. elpais.com/sociedad/2013/03/01/actualidad/1362165444_533165.html

Para Guillermo Cullel, experto en periodismo digital, citado por James Breiner, en el artículo titulado Emprendimientos periodísticos, publicado en la Revista Mexicana de Comunicación, los periodistas independientes en Internet tienen la posibilidad de liberarse de los poderes, las prácticas y pensamientos que han definido a la prensa tradicional. "Estos nuevos medios representan un desafío a los grandes poderes tradicionales de América Latina, es decir, a la oligarquía del poder político, económico y mediático que han controlado el destino de muchos países en una u otra forma" Breiner, James (2013) Emprendimientos periodísticos. Revista Mexicana de Comunicación. Recuperado de www.mexicanadecomunicacion.com.mx.

Muchos medios digitales en Latinoamérica han nacido concebidos bajo este pensamiento. Plaza Pública de Guatemala, por ejemplo, se dedica a indagar lo que ocurre en las altas esferas del poder. La página logró un acuerdo con Wikileaks para obtener los documentos secretos sobre su país, revelados por Julian Assange; ese material les sirvió de insumo para escribir los perfiles de los candidatos de las últimas elecciones. Tiene unas 90.000 visitas al mes y 50.000 usuarios únicos. Ese enfoque también está presente en Animal Político y Reporte Índigo de México, en El Confidencial de Nicaragua y en Verdad Abierta y la Silla Vacía de Colombia. En el sur del continente, la tendencia es la misma. En Perú está IdI Reporteros, Ciperchile de Chile, en Argentina Puercoespín y Chequeando y en Brasil Apublica.

Otro ejemplo de lo que se hace en la Región es Radio Ambulante, aunque su enfoque no es la investigación, sino el 
contar historias humanas provenientes de toda Latinoamérica.

"La comunidad de periodistas en Latinoamérica tiene hambre de un nuevo periodismo independiente y necesita, ante todo, capacitación en nuevas habilidades y tecnologías. La revolución de los medios está destruyendo los modelos de negocio tradicionales, pero empieza a creer oportunidades para quienes tienen la capacidad de cambiar y adaptarse". Quesada, Juan Diego (2013, 1 de marzo) El "boom" de la prensa digital latinoamericana. Diario El País. Recuperado http://sociedad.elpais.com/sociedad/2013/03/01/actualidad/1362165444_533165.html

Los medios de Latinoamérica han demostrado su capacidad de adaptación, evolución y reproducción, de ahí que a la lista de medios agregada, se suman muchos más. Resulta complicado precisar una cifra de cuantos nuevos medios existen en la Región, ya que no hay muchos estudios especializados y actualizados. Además, constantemente, aparecen nuevos proyectos por lo que es muy difícil contar con una lista actualizada. Según un estudio realizado por la Universidad de Florida y el profesor investigador Ramón Salavarría, experto en Periodismo digital, titulado Regenerating Journalism: Exploring the Alternativeness and Digital-ness of on line Native Media in Latin America, publicado en el 2015, hasta septiembre 2015 se registraron 67 medios nativos digitales en 18 países de lengua española y portuguesa en América Latina. Salavarría, Ramón (2015, 22 de mayo) Radiografía de los medios digitales en América Latina. Portal las2orillas. Recuperado de http://www.las2orillas. co/radiografia-de-los-medios-digitales-en-america-latina/.

Aquí cabe hacer una puntualización importante respecto al término medios nativos digitales. Este se usa para referirse a los medios que han nacido en el entorno digital y que no tienen una versión offline que les anteceda. En ese sentido, este estudio se enfoca en este tipo de medios, exclusivamente. En este trabajo, además, se revela cuales son los medios con mayor influencia en la Región; encabezando la lista está 14 y medio, diario cubano. En décimo y undécimo puesto aparecen dos medios ecuatorianos: Ecuador en vivo y Ecuador inmediato, respectivamente. Salavarría, Ramón (2015, 22 de mayo) Radiografía de los medios digitales en América Latina. Portal las2orillas. Recuperado de http://www.las2orillas.co/radiografia-de-los-medios-digitales-en-america-latina/.

Sin duda, los emprendimientos periodísticos son una tendencia, relativamente nueva, que ha transformado la forma de entender el periodismo y que cada día suma más seguidores. Según 
recoge una nota de diario El País, titulada El "boom" de la prensa digital latinoamericana, Daniel Moreno director del Portal Animal Político de México, señala que una de las razones de porque la gente está prefiriendo estos medios es porque, de alguna manera, son cercanos a las personas al mostrar los temas que a estas, verdaderamente, les interesan y les preocupan, ya que por la naturaleza del medio -interactiva y participativa-, están, permanentemente, recibiendo retroalimentación de sus usuarios. "Estos medios saben escuchar a sus lectores. Prueba enviar un tuit a los grandes medios. No te van a contestar, no hay nadie del otro lado". Quesada, Juan Diego (2013, 1 de marzo) El "boom" de la prensa digital latinoamericana. Diario El País. Recuperado http://sociedad.elpais.com/sociedad/2013/03/01/actualidad/1362165444_533165.html

Una de las tendencias que ha nacido, precisamente, basada en la necesidad de mantener cercanía con la audiencia, es el periodismo hiperlocal. En todo el continente existen emprendimientos periodísticos concebidos bajo este enfoque, mismo que da cuenta de lo que ocurre en el entorno más cercano, una parroquia, un barrio, o una zona muy reducida geográficamente. "La independencia de estos medios les hace más ágiles y transparentes, el contexto geográfico reducido les facilita cubrir hasta casi el cien por ciento de la información de interés local, siendo además un buen recurso como fuente de información para medios más grandes que necesitan de información local". (Yuste, Cabrera 2014). "En cuanto a la temática, los medios tradicionales dedican poco espacio a los temas importantes de la vida cotidiana de la gente de a pie, tales como la economía familiar, la educación, la salud, el medio ambiente, entre otros. Hay brechas enormes en la oferta de información y hay segmentos de la población mal atendidos por los grandes medios. Esta es la oportunidad para que nuevos medios digitales ofrezcan contenidos que sirvan a comunidades definidas por geografía - un barrio, una aldea, un país- o por interés compartidos". Breiner, James (2013) Emprendimientos periodísticos. Revista Mexicana de Comunicación. Recuperado de www.mexicanadecomunicacion.com.mx.

Otro factor que influiría en el hecho de que las audiencias se estén trasladando masivamente a este tipo de medios, según Daniel Moreno, es que gran parte de quienes navegan por las redes sociales e Internet, en general, son los jóvenes; quienes ahora tienen mucho interés por estar informados. Según un estudio de Comscore, compañía de investigación de marketing en Internet, Latinoamérica es la región más involucrada en redes sociales a nivel global. Quesada, 
Juan Diego (2013, 1 de marzo) El "boom" de la prensa digital latinoamericana. Diario El País. Recuperado http://sociedad. elpais.com/sociedad/2013/03/01/actualidad/1362165444_533165.html

\section{Emprendimientos periodísticos en Ecuador}

Ecuador no ha quedado fuera de esta nueva forma de hacer periodismo. Según el libro Ciberperiodismo en Iberoamérica que recoge la historia del nacimiento y evolución de los medios digitales en América Latina, España y Portugal, el primer medio nativo digital del país fue Ecuador Inmediato, mismo que vio la luz en el 2004. Redacción Quito (2016, 23 de febrero) Ecuadorinmediato.com es destacado en libro de historia sobre veinte primeros años del periodismo digital en Iberoamérica. Portal Ecuadorinmediato. Recuperado de http://www. ecuadorinmediato.com/index.php?module $=$ Noticias\&func $=$ news user view\&id=2818796907. Asimismo, en el estudio titulado Mapa de medios digitales en el Ecuador, publicado en el 2012 por CIESPAL, se mencionan cuáles fueron los primeros medios digitales provenientes de medios tradicionales. En esta categoría se encuentran Diario "El Comercio" y Diario "Hoy", los que entre 1995 y 1996 desarrollaron incipientes versiones digitales, mismas que eran una copia de la versión impresa, es decir, en ellas aún no estaban presentes los elementos propios del lenguaje digital. Rivera, José (2012). Mapa de medios digitales del Ecuador 2012. Blog sobre Comunicación y "marketing" Digital Tikinauta. Recuperado de tikinauta.com/blog. Según los conocedores de la evolución de los medios digitales en el Ecuador, Diario "Hoy" fue el primer periódico digital de Sudamérica. Estos antecedentes muestran que el país tiene una historia de más de dos décadas en este campo, por lo que no es raro que en los últimos años hayan surgido más emprendimientos periodísticos digitales.

El estudio presentado en el 2012 por CIESPAL es uno de los más completos y consolidados, por lo que se le ha tomado como referencia para aproximarse al entorno de los medios nativos digitales en el país, aun cuando no sea actualizado. Como se dijo anteriormente, los estudios en este campo, difícilmente cuentan con datos actualizados, ya que cada día nace un nuevo medio. En el país los estudios son muy escasos, algunos de los más actuales, precisamente, han tomado al de CIESPAL como referente para analizar y comparar cómo ha evolucionado esta industria, tomando en cuenta que entre el 2010 y el 2012, se dio un despunte en la creación de emprendimientos periodísticos, según un estudio de Fundamedios, publicado en el 2015, sobre el que se hablará más adelante. 
De acuerdo con lo que arroja el estudio de CIESPAL, hasta el 2012 existían 34 medios nativos digitales: 16 periódicos, 14 radios y 4 revistas. Las provincias con mayor cantidad de medios digitales eran: Pichincha, Guayas, Azuay, Chimborazo, Manabí, Loja, Cañar y El Oro. En Napo, Orellana, Zamora y Carchi no se registraron este tipo de medios Rivera, José (2012). Mapa de medios digitales del Ecuador 2012. Blog sobre Comunicación y "marketing" Digital Tikinauta. Recuperado de tikinauta.com/ blog. Sin embargo, tres años más tarde las cifras cambiaron, lo cual quedó recogido en una investigación realizada por Fundamedios y publicada en el 2015, misma que se puede considerar como la investigación publicada más reciente y actualizada. Según esta, a abril del 2015 en Ecuador habrían 60 medios nativos digitales, considerados emprendimientos periodísticos. A continuación se presentan los datos más reveladores del estudio. La fuente de estos datos fue Fundamedios, quien facilitó a la autora de este artículo los siguientes gráficos.

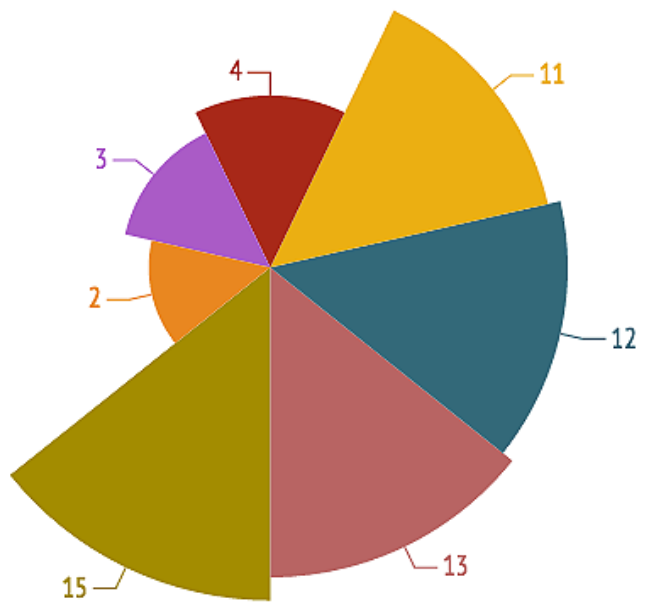

Radios Medios regionales Periódico/agregadores noticias Especializados \begin{tabular}{l|l|l}
\hline De investigación De periodismo narrativo Opinión &
\end{tabular}

Figura 1. Tipos de medios que existen. 


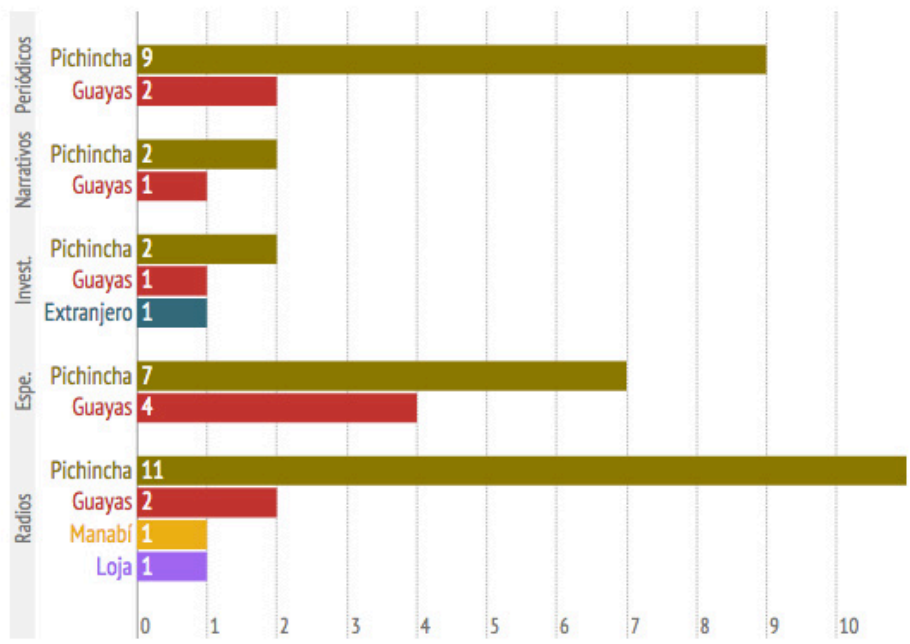

Figura 2. Tipos de medios por provincia.

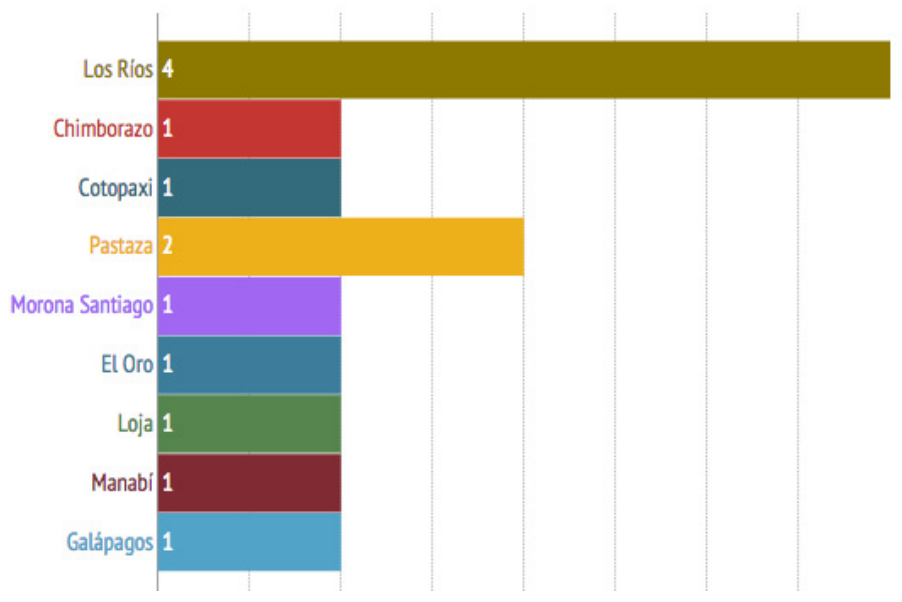

Figura 3. Número de medios por provincia. 


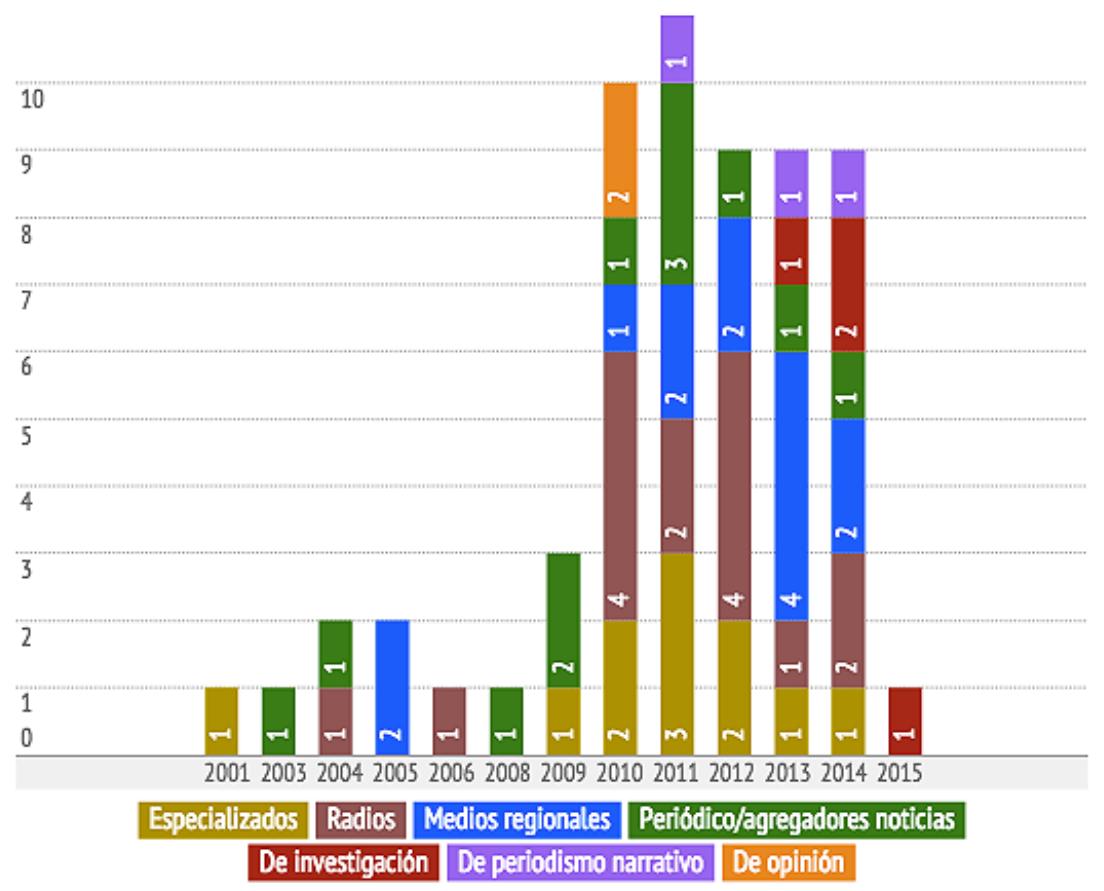

Figura 4. La evolución de los medios en los últimos años.

Tomando los datos de este estudio y comparando con las cifras que arrojó el estudio de CIESPAL, se ve que los emprendimientos periodísticos aumentaron de 34 a 60 en tres años -del 2012 al 2015- registrando, además, que entre el 2010 y el 2012 fue el período en el que más medios se crearon.

En el último tiempo, la crisis de las empresas informativas se ha agudizado; se han generado más despidos y el gobierno ha dificultado la labor de los medios con la puesta en marcha de la Ley Orgánica de Comunicación aprobada en el 2013. Según señala una nota de Diario "El Comercio", en el 2015 la SUPERCON dictó 299 sanciones a medios de comunicación entre multas económicas, pedidos de réplicas y rectificaciones. Guerrero, Ana (2015, 24 de junio) La SUPERCOM sancionó a 198 medios en dos años. Diario El Comercio. Recuperado en http://www.elcomercio.com/actuali$\mathrm{dad} /$ supercom-sanciones-medios-leydecomunicacion.html 
Siguiendo la tendencia que ha estado presente en otros países del continente, en Ecuador muchos de los emprendimientos periodísticos que se han creado, han nacido como espacios para dar cuenta de lo que sucede en las esferas de poder y se han posicionado como medios de investigación y denuncia. Estos son PlanV, La Historia, Focus Ecuador y Milhojas.

Desde que entrara en vigencia la Ley de Comunicación, e incluso desde mucho antes, varios periodistas han sido perseguidos por el gobierno por lo que han dejado sus puestos en los medios tradicionales y han optado por crear sus emprendimientos, en algunos casos, obligados por la situación. Ese es el caso de Martín Pallares, periodista político de Diario "El Comercio", quien tras varias publicaciones en su cuenta personal de Twitter en contra del gobierno, fue despedido del periódico, aduciendo el no haber frenado sus comentarios, luego de reiterados pedidos de que lo haga. A partir de este hecho abrió su sitio Martinpallares.net.

El cierre del Diario "Hoy" en el 2014, acontecido como lo explicó su director, por la crisis del sector, dejó en el desempleo a los periodistas José Hernández y Roberto Aguilar, quienes meses después abrieron sus blogs, Sentido Común y Estado de Propaganda, respectivamente, desde donde cuestionan al Régimen.
Tras la oposición que recibieron estos espacios por parte del gobierno, decidieron unirse para fortalecer su tarea periodística y es así como crearon el portal Cuatropelegatos, junto a Martín Pallares y Crudo Ecuador, espacio de Memes críticos en contra del gobierno.

Otro de los elementos que hace al entorno digital terreno fértil para los emprendimientos periodísticos es que la Ley Orgánica de Comunicación no puede regular los contenidos que circulan por este. Por esa razón, quienes han querido seguir ejerciendo la profesión desde un espacio menos censurado, han optado por este camino. Otra ventaja de lo digital es que permite emprender sin una mayor inversión; crear un medio de comunicación propio, en relación con el costo, no tiene comparación con lo que en el pasado representaba abrir un canal de televisión o un periódico.

Siguiendo con los datos revelados por el estudio de Fundamedios, hasta el 2015 se registraron 15 radios digitales, evidenciando un ligero aumento de una radio, en relación con la investigación presentada por CIESPAL que registró 14 en el 2012. Esto podría deberse a que por la naturaleza propia del medio, trasladarla al entorno digital no resulta muy complicado, además al hecho que las audiencias en general, se han trasladado masivamente al Internet. Es importante también destacar 
que es posible que los emprendedores se sientan más confiados de invertir en este tipo de medio porque, actualmente, existe más claridad sobre la rentabilidad que pueden generar; los modelos de negocio cada vez están más afinados. Wambraradio, Rayuela Radio, Radio Cocoa y Flacso Radio son algunos de los proyectos más destacados. Radio Cocoa y Flacso Radio, espacios creados desde la Universidad San Francisco de Quito y la Facultad Latinoamericana de Ciencias Sociales, FLACSO, respectivamente, no estarían enmarcados bajo el concepto de emprendimiento propiamente, ya que al pertenecer a estas universidades, tendrían otro enfoque. Sin embargo, se los ha incluido en esta lista ya que han surgido en el entorno digital como proyectos alternativos, nuevos medios, distintos a las radios tradicionales.

El estudio muestra que luego de las radios, los emprendimientos que más han crecido son los medios locales. Esto podría sugerir que la crisis también ha golpeado duramente a las empresas informativas de varias ciudades del país, por lo que muchos periodistas han optado por desarrollar estos proyectos. Se puede pensar, además, que el concepto de periodismo hiperlocal cada vez está entendiéndose mejor y que está siendo aplicado en más emprendimientos. Llama la atención el aparecimiento de este tipo de medios en la provincia de
Los Ríos, que según este estudio registra cuatro. Mientras que en el 2012, según el estudio de CIESPAL, no registraba ninguno. Ciertamente, el emprendimiento periodístico se ha convertido en una alternativa presente, incluso, en ciudades pequeñas, lo que evidencia que es una tendencia que va en aumento en todo el país. En el caso de esta provincia, el diario digital Aldía es uno de los proyectos más representativos, mismo que informa sobre lo que sucede en Quevedo y Babahoyo, principalmente. Otras iniciativas ubicadas en distintas ciudades son: Cotopaxinoticias, Expresatemoronasantiago, Quevedoaldia, Machalamovil y Galapagosdigital.

Tanto el informe de CIESPAL como el de Fundamedios, coinciden en que las provincias en las que existen mayor cantidad de emprendimientos periodísticos son Guayas y Pichincha. Son, precisamente, en estas provincias donde más se han creado medios enfocados a la narrativa y a la investigación periodística. En ese sentido, y hablando en términos de géneros periodísticos, en estos medios se estarían desarrollando los géneros de la crónica y el reportaje a profundidad, considerados de mayor complejidad, en relación con el género de la noticia, por ejemplo, que, principalmente, se desarrolla en los periódicos y medios agregadores de noticias. Esto tiene que ver con que, tradicionalmente, 
en Quito y Guayaquil el periodismo se ha consolidado con mayor fuerza y se ha especializado más que en otras ciudades del país. Aquí se hace un periodismo que puede definirse como de mayor calidad, en relación con otros lugares, y eso está demostrado en el tipo de emprendimientos periodísticos de estas dos ciudades, en las que se ha optado por hacer medios no solo noticiosos, sino con un contenido periodístico más variado y más trabajado. Algunos de estos son: labarraespaciadora, Gkillcity y lamalaletra.

Cabe destacar que en Pichincha, concretamente en Quito, se encuentran dos medios ubicados en décimo y undécimo lugar en la lista de los medios digitales más influyentes de la Región, según el estudio Regenerating Journalism: Exploring the Alternativeness and Digital-ness of on line Native Media in Latin America, realizado por la Universidad de Florida y el investigador Ramón Salavarría; estos son los portales noticiosos Ecuador Inmediato y Ecuador Envivo. Otros espacios de noticias son: La Republica, Periodismo Ecuador y Confirmado.net. Cabe señalar que en este artículo solo se mencionan algunos de los emprendimientos periodísticos que existen, los mismos que fueron escogidos por su nivel de representatividad.

\section{Modelos de negocio}

El aumento de los emprendimientos digitales en toda la Región y en el país es un hecho. Lo que también es un hecho, y que resulta paradójico, es que a pesar de que muchos periodistas han optado por este tipo de proyectos para generar ingresos que les permitan vivir, como con cualquier otro empleo, siguen sin descubrir la fórmula de cómo hacerlo. Si bien algunos proyectos aportan réditos económicos importantes a sus creadores, hay otros que todavía siguen experimentando con distintos modelos de negocio, sin generar resultados. Nadie tiene la fórmula de cómo rentabilizar al máximo un emprendimiento periodístico.

Según un estudio de la Fundación Nuevo Periodismo FNPI, el 57\% de los emprendimientos de la Región genera pérdidas; solo el 18,5 \% genera ingresos mayores a sus costos. Quesada, Juan Diego (2013, 1 de marzo) El "boom" de la prensa digital latinoamericana. Diario El País. Recuperado http://sociedad.elpais.com/sociedad/2013/03/01/actualidad/1362165444_533165.html

Tomando en cuenta esta realidad, la Fundación creó un Laboratorio de Emprendimientos Periodísticos Digitales para apoyar a los emprendedores en su afán de lograr la sostenibilidad financiera de sus proyectos. El objetivo de este 
espacio es capacitar a los periodistas en temas como: tecnología, mercadeo, estrategia comercial, mercadeo de medios, como vender publicidad, nuevas fuentes de ingreso para medios y cómo dirigir una empresa. Breiner, James (2013) Emprendimientos periodísticos. Revista Mexicana de Comunicación. Recuperado de www.mexicanadecomunicacion. com.mx. De esta forma, la Fundación contribuye a fortalecer el sector de los emprendimientos periodísticos en el continente y a apoyar el buen periodismo independiente.

En Ecuador la situación es parecida a la de otros países. En un reportaje publicado por la Revista "Vistazo" titulado El nuevo periodismo on line, el periodista Martín Pallares afirma que aunque su portal Martinpallares.net tiene 4.000 lectores al día, no recibe ningún rédito económico. En iguales circunstancias se encuentra el periodista Juan Carlos Calderón de Plan $V$, quien afirma que el portal genera lo mínimo para pagar solo a tres colaboradores: un redactor, un diseñador y un fotógrafo. A Plan V le faltan recursos pero le sobran colaboradores quienes escriben gratuitamente. "Hay un montón de buenos periodistas en la desocupación, mucha gente valiosa en circunstancias complicadas. Eso disminuye la calidad y el impacto del periodismo a la larga", señala. Santos, Tristana (2015, 12 de octubre) El nuevo periodismo on line.
Revista Vistazo. Recuperado en http:// vistazo.com/seccion/pais/el-nuevo-periodismo-online

Plan $V$ recibe una subvención de la Fundación Nacional para la Democracia, entidad vinculada al gobierno estadounidense. Ha intentado vender publicidad, lo cual no ha resultado muy efectivo, por lo que ha recurrido al "crowfunding", según señala el reportaje de Revista "Vistazo".

Uno de los grandes limitantes que tienen los emprendimientos periodísticos es la cultura de la gratuidad en la que todos estamos insertos con la llegada de la era digital. ¿Por qué pagar por un contenido que puede ser consumido gratuitamente? En ese sentido, el principal reto de estos medios es generar un contenido especializado, que no se encuentre en ningún otro sitio para que tenga la posibilidad de ser comercializado.

Precisamente, en esto se basa uno de los tantos modelos de negocio que son aplicados por estos medios, se trata del modelo de venta de contenido, bajo el cual el medio vende toda la información que produce o parte de ella. En la medida que la información sea más especializada, puede despertar el interés de pagar por ella.

Para los nuevos medios, la aplicación de uno u otro modelo de negocio ha sido un ejercicio de prueba y error 
permanente. Cada uno ha probado con el modelo que más se ajuste a sus necesidades, e incluso, han intentado combinando varios. En algunos casos los resultados son más efectivos que en otros.

El modelo más usado en la mayoría de los emprendimientos ecuatorianos, sigue siendo el de venta de publicidad. El crowfunding, es otra de las alternativas para recaudar dinero; este consiste en recibir colaboraciones de los lectores fieles quienes, voluntariamente, aportan para asegurar la permanencia del sitio. El patrocinio también es utilizado, este consiste en que una marca $u$ organización patrocine el sitio o una sección de este, entregando dinero; a cambio de esto en el medio habrá presencia de marca. Las opciones de modelos de negocios son muchas, otra son las suscripciones, que al igual que como funcionan en la prensa escrita, el lector paga por adelantado para recibir una cantidad específica de ediciones. Otra alternativa es la venta de contenidos a la medida, o lo que se conoce como "marketing" de contenidos. Se trata de generar contenidos de calidad para una marca en concreto. En este caso, los contenidos pasan a ser de la marca, la que los utilizará de la forma que desee. Entre las opciones menos usadas en nuestros medios y menos convencionales están la venta de cupones, a través de la cual el medio gana al ser el intermediario entre la marca y el consumidor. El medio recibe un porcentaje de las ventas de los cupones. Otra es la creación de contenidos para tabletas y teléfonos. Este modelo plantea la creación de información específica para estos dispositivos; la ganancia está en que los usuarios compran la información que se adapta a sus dispositivos. Por último, está la venta de productos de todo tipo, la organización de eventos y la consultoría. (Yuste, Cabrera 2014). Sea cual sea el modelo, todos le apuntan a develar el secreto del éxito comercial, que, por ahora, permanece oculto para muchos emprendedores.

El emprendimiento periodístico como tendencia actual, no solo se manifiesta en los proyectos mencionados, sino también en el enfoque que hoy se le está dando al periodismo dentro de las aulas universitarias. La visión sobre el oficio periodístico ha cambiado, de pasar a pensar en el periodista como un asalariado de las grandes empresas informativas, a ser el dueño de su propio medio. Hoy el periodista se construye como una marca de credibilidad independiente, quien desde un espacio creado bajo los lineamientos del emprendimiento, está ejerciendo un nuevo periodismo, en una época en el que el oficio periodístico y los medios de comunicación se están reinventando.

Este pensamiento se hace tangible en los emprendimientos periodísti- 
cos que son diseñados como parte de la materia Dirección de Empresas Informativas que se imparte en la carrera de Comunicación con mención en Periodismo para prensa, radio y televisión de la PUCE. Desde este espacio, se motiva en los estudiantes a crear su propio medio y se les guía en su construcción. Además, se les ayuda a comprender las transformaciones que ha experimentado la profesión, fruto de la nueva era informativa en que vivimos; a desechar la idea del periodista como una figura de poca autonomía, cuya valía se da en función del medio al que pertenece y a reconocerse como periodistas autónomos, quienes se forjan a sí mismos como marca de credibilidad y calidad. Aunque todavía no hay resultados concretos, que hayan trascendido fuera del aula; la idea de creer que es posible un periodismo nuevo ya está interiorizada. Ese es el primer paso.

\section{CONCLUSIONES}

La dificultad más grande que enfrentan los emprendedores es el encontrar la forma de rentabilizar su proyecto. En la mayoría de los casos, estos perciben ingresos mínimos, y en otros, ninguno, por lo que su gran desafío sigue siendo identificar el mecanismo que les permita sacar la mayor ventaja económica. En esa lucha, cada uno ha planteado un modelo de negocio propio que se ajusta a su realidad, de ahí que existan tantos modelos y que respondan de manera distinta al objetivo que todos tiene en común: ganar dinero.

En el país existen pocos estudios actualizados sobre emprendimientos periodísticos, sin embargo, más allá de las cifras, la percepción es que existen muchos pero que no están posiciona- dos y, por lo tanto, no se conocen. Es complicado desarrollar un estudio consolidado ya que, permanentemente, aparecen y desaparecen medios, por lo que se considera que en el país, este es todavía un sector inestable.

Al igual que en otros países de la Región, en el Ecuador muchos emprendimientos han nacido con un enfoque que plantea un periodismo renovado, fresco, independiente de las estructuras formales de los medios de comunicación tradicionales. Desde esa perspectiva, son considerados por las audiencias como espacios creíbles y confiables, por lo que han puesto en duda la credibilidad de los medios tradicionales, los mismos que antes eran incuestionables y eran los protagonistas absolutos de la opinión pública. 
En el caso del Ecuador, a la revolución digital y a la crisis económica global, se ha sumado otro factor importante que ha impulsado el emprendimiento: la relación medios-gobierno. La posición del gobierno de debilitar la libertad de expresión ha limitado a muchos periodistas, quienes han optado por seguir haciendo su trabajo desde un espacio menos censurado y más libre: el digital.
El emprendimiento periodístico es visto como una salida a la crisis mundial de las empresas informativas, y como una muestra clara de que estamos en una era distinta del periodismo, en la que el medio en sí ya no es el protagonista, sino el periodista y su capacidad de adaptación a un escenario nuevo que, al menos en el Ecuador, todavía no termina de entenderse. 


\section{BIBLIOGRAFÎA}

Donado V., Donaldo A. (2005) De la información a la opinión: los géneros periodísticos. Bogotá. Magisterio

Yuste Bárbara, Cabrera Marga (2014) Emprender en periodismo: nuevas oportunidades para el profesional de la información. Barcelona. Editorial UOC.

Breiner James (2013) Emprendimientos periodísticos en Revista Mexicana de Comunicación http://mexicanadecomunicacion.com.mx/ rmc/

La inversión publicitaria decae en medio de la irrupción digital en Revista Líderes http://www.revistalideres. ec/lideres/inversion-publicitaria-digital-ecuador.html

La publicidad cae el 13,9\% en los medios tradicionales, pero aumenta en los sitios web en Diario El Telégrafo._http://www.eltelegrafo.com. ec/noticias/medios/1/la-publicidad-cae-el-13-9-en-los-mediostradicionales-pero-aumenta-ensitios-web

Con un record negativo de 377 agresiones, 2015 cierra como el peor año para la libertad de expresión en Ecuador en Portal Juicio Crudo http://www.juiciocrudo.com/ articulo/con-un-record-negativo-de-377-agresiones-2015-cie- rra-como-el-peor-ano-para-la-libertad-de-expresion-en-el-ecuador/5206

INEC publica cifras del mercado laboral de septiembre de 2016 en Portal de Instituto Ecuatoriano de Estadísticas y Censos http://www. ecuadorencifras.gob.ec/inec-publica-cifras-del-mercado-laboral-de-septiembre-2016/

Los puestos de trabajo se reducen en una coyuntura económica difícil en Diario El Comercio http:// www.elcomercio.com/actualidad/desempleo-trabajadores-crisis-economia-empresas.html

Televisora privada Ecuavisa despide a 40 trabajadores para bajar costos en Agencia de Noticias Andes http:// www.andes.info.ec/es/noticias/ televisora-privada-ecuavisa-despide-40-trabajadores-bajar-costos.html

El boom de la prensa digital latinoamericana en Diario El País http://sociedad.elpais.com/ sociedad/2013/03/01/actualidad/1362165444_533165.html

Radiografía de los medios digitales en América Latina en Portal las2orillas http://www.las2orillas.co/ radiografia-de-los-medios-digitales-en-america-latina/ 
Ecuadorinmediato es destacado en libro de historia sobre veinte primeros años del periodismo digital en Iberoamérica en Portal Ecuador Inmediato http://www.ecuadorinmediato.com/index.php?mo$\underline{\text { dule }=\text { Noticias\&func }=\text { news user }}$ view\&id=2818796907.

Hay más medios nativos digitales pero su sostenibilidad es difícil en Diario El Comercio http://www. elcomercio.com/actualidad/medios-nativos-digitales-sostenibilidad-publicidad.html
El nuevo periodismo on line en Revista Vistazo http://vistazo.com/ seccion/pais/el-nuevo-periodismo-online

Ciespal (2012) Estudio: Mapa de medios digitales de Ecuador http:// tikinauta.com/blog/wp-content/ uploads/2014/09/Mapa-medios-digitales-José-Rivera-Costales.pdf.

Fundamedios (2015) Estudio: Mapeo de emprendimientos periodísticos en Ecuador.

\section{REFERENCIAS BIBLIOGRÁFICAS}

Pérez Colome Jordi (2012) Microperiodismos digitales: aventuras en tiempos de crisis I, editorial UOC, Barcelona.

Pérez Colome Jordi (2014) Microperiodismos digitales: aventuras en tiempos de crisis II, editorial UOC, Barcelona

FJ Paniagua Rojano, M Gómez Aguilar, ME González Cortés (2014) Incentivar el emprendimiento periodístico desde la Universidad en Revista Latina de Comunicación Social.
Breiner James (2014) Emprendimientos periodísticos digitales en todo el mundo en Blog Periodismo emprendedor en Latinoamérica.

Franco Marta (2014) Optimismo para periodistas: claves para entender los nuevos medios de comunicación, editorial UOC, Barcelona.

Casero Ripollés Andreu (2013) Periodismo emprendedor: estrategias para incentivar el autoempleo periodistico como modelo de negocio en Revista Cientíica Complutense 
